\title{
Lamotrigine extends lifespan but compromises health span in Drosophila melanogaster
}

\author{
Agnesa Avanesian · Behnood Khodayari • \\ Jeffery S. Felgner · Mahtab Jafari
}

Received: 3 September 2008/Accepted: 13 April 2009/Published online: 9 May 2009

(C) The Author(s) 2009. This article is published with open access at Springerlink.com

\begin{abstract}
The discovery of life extension in Caenorhabditis elegans treated with anticonvulsant medications has raised the question whether these drugs are prospective anti-aging candidate compounds. The impact of these compounds on neural modulation suggests that they might influence the chronic diseases of aging as well. Lamotrigine is a commonly used anticonvulsant with a relatively good adverseeffects profile. In this study, we evaluated the interaction between the impacts of lamotrigine on mortality rate, lifespan, metabolic rate and locomotion. It has been proposed in a wide range of animal models that there is an inverse relationship between longevity, metabolic rate, and locomotion. We hypothesized that the survival benefits displayed by this compound would be associated with deleterious effects on health span, such as depression of locomotion. Using Drosophila as our model system, we found that lamotrigine decreased mortality and increased lifespan in parallel with a reduction in locomotor activity and a trend towards metabolic rate depression. Our findings underscore the view that
\end{abstract}

A. Avanesian · M. Jafari ( $\square)$

Department of Pharmaceutical Sciences, University of California, Irvine, Irvine, CA 92697, USA

e-mail: mjafari@uci.edu

B. Khodayari · J. S. Felgner

Undergraduate Program, School of Biological Sciences, University of California, Irvine, Irvine, CA 92697-2525, USA assessing health span is critical in the pursuit of useful anti-aging compounds.

Keywords Lamotrigine Drosophila .

Lifespan · Health span · Mortality ·

Metabolic rate $\cdot$ Locomotion

\section{Introduction}

Mutations in genes such as age-1 (Arking 1998), daf2 (Kenyon et al. 1993; Kimura et al. 1997), Indy (Rogina et al. 2000), and chico (Clancy et al. 2001) have been found to increase life span in the laboratory organisms Caenorhabditis elegans and Drosophila melanogaster. Genetic manipulations offer helpful clues in identifying pathways for pharmacological interventions, but there is the possibility that these genetic manipulations result in adverse metabolic effects. In particular, there is the possibility that "longevity mutants" increase lifespan only because of a "refrigeration" effect. The "refrigeration" effect refers by analogy to the phenomenon in which an increase in lifespan is seen at environmentally low temperatures due to decreases in metabolic rate (Van Voorhies et al. 2006). The inverse correlation between metabolic rate and longevity in Drosophila has been reported in a number of studies. It has been suggested that poikilotherms can have their lifespan 
extended or curtailed as their metabolic rates are decreased or increased, respectively (Finch 1990; Rose 1991). In a recent study, Van Voorhies et al. (2008) evaluated $\mathrm{CO}_{2}$ production in a large set of recombinant inbred Drosophila males at various ages. Although they reported a wide range of longevities and metabolic rates, no correlations between the two variables were observed. This contrasts with earlier experimental results, suggesting significant complexity in the relationship between lifespan and metabolic rate. As humans are homeotherms with a fairly stable metabolic rate, drugs that act via substantial lowering of metabolic rate, thus producing hypo metabolic syndromes, may not be appropriate candidates for anti-aging interventions. Poikilothermic animal models, such as C. elegans and Drosophila, have the ability to tolerate metabolic rate depression well during phases of poor environmental conditions or deprivation of nutrients (Klass et al. 1983; Van Voorhies and Ward 1999). Since humans do not posses the same capacity to endure such depression of their metabolism, it is necessary to evaluate metabolic rate as a confounding factor; especially when considering results that might be extrapolated to the clinical context.

Other potential confounds, such as fecundity depression, can also result in lifespan extension. These factors should also be evaluated during an antiaging substance screening process (Jafari and Rose 2006). Some compounds may increase lifespan simply by substantially depressing fecundity; a substantial depression in fecundity alone is sufficient to increase longevity in Drosophila (Smith 1959). In order to ensure that the inferred anti-aging properties are a direct effect of the compound, and not adverse physiological changes, we need to assess the impact of the compound on other aspects of the organism's physiology. A number of studies in the anti-aging literature have addressed such secondary effects by evaluating the impact of the proposed anti-aging compound on fecundity and metabolic rate (Kang et al. 2002; Jafari et al. 2007a, b, 2008). For instance, Rhodiola rosea increased Drosophila lifespan at certain doses but the fecundity was also reduced at those doses (Jafari et al. 2007a). Therefore, a fecundity assay is an important check for artifactual lifespan enhancement.

In addition to checking for the impact of compounds on physiological confounds of aging, we should also evaluate their effect on biomarkers of health span such as locomotor activity. In 1987, Le Bourg reported an association between locomotor activity and longevity in Drosophila. He showed that spontaneous locomotor activity declined in females as they aged and increased in males up to week five and then began to decrease (Le Bourg 1987). In another recent study, locomotion declined with aging in Drosophila although males performed better than females at advanced age (Simon et al. 2006).

Our study evaluated the impact of lamotrigine on lifespan, physiological confounds of aging, and health span of D. melanogaster. Although the mechanism of action of lamotrigine has yet to be fully understood, it is believed that the drug primarily targets voltage-sensitive sodium channels that inhibit neural excitation through the repression of the neurotransmitters glutamate and aspartate (Lang et al. 1993). These neurotransmitters play key roles in numerous physiological activities of various species (Rock 1968; Fagg and Foster 1983; Wroblewski and Danysz 1989; Advokat and Pellegrin 1992; Navarro et al. 1998; Burton and Kazemi 2000). Drosophila possesses glutamate neurotransmitters that are regulated by sodium channels in their nervous system. Studies conducted on the anesthetic drug isoflurane have shown that Drosophila larvae exhibited decreased glutamate neurotransmitter release when treated with the drug. This decrease in neuronal firing is paralleled with a sedative phase where decreased locomotor activity was observed (Sandstrom 2004), suggesting a conserved neuronal physiology shared by vertebrates and arthropods.

Due to lamotrigine inhibitory actions on the glutamate neurotransmitter and Drosophila's conserved neural physiology with respect to the glutamate receptor, relative to mammals, we hypothesized that a depression of metabolic rate and locomotor activity would be observed in flies supplemented with the drug.

\section{Materials and methods}

\section{Drosophila population employed}

All D. melanogaster stocks used in these experiments were ultimately derived from a sample (called "IV") of the Amherst, Massachusetts, Ives population that 
was collected in 1975 and cultured at moderate to large population sizes ever since (Rose et al. 1984). This population has been reared at controlled densities (50-80 eggs per vial) for more than 700 generations with discrete generations cultured every 2 weeks.

\section{Drug administration}

Fruit flies were supplemented with lamotrigine (GlaxoSmithKline) at 1, 3, 6, 12, 24, and $48 \mathrm{mg} / \mathrm{ml}$, alongside controls that were handled in the same manner but not exposed to lamotrigine. These doses reflect the final concentration of lamotrigine in the yeast paste that was supplemented to adult flies during the experiment. Stock solutions were prepared by dissolving lamotrigine powder in water.

\section{Mortality assay}

All flies used in these assays were raised as larvae in $5 \mathrm{ml}$ of standard banana-molasses food at densities between 50 and 80 eggs per 8 -dram vial. Populations were maintained at about $25^{\circ} \mathrm{C}$ with constant illumination. During the assays, adults were kept in standard 8-dram vials containing $5 \mathrm{ml}$ of bananamolasses food and coated with $75 \mu$ of the drugyeast paste mixture. Adults were transferred to fresh vials and survivors were counted every 2 days over a 28 day period. For each dose, 320 males and 320 females were exposed to the compound. There were 4 males and 4 females in each vial, with a total of 80 vials per dose per sex, and the flies were transferred every other day during the aging phase (4 weeks for IV stocks). Data were analyzed by examining the number of surviving flies at the end of week four. The statistical evaluation at the end of week four is based on data accumulated from the records of survivors obtained every 2 days. Significant differences between the control and each drug treatment were assessed using the Pearson chi-square test. Mortality assays were replicated for doses that decreased the mortality rate. These doses were subsequently used in the lifespan assay.

\section{Lifespan assay}

One hundred sixty male and 160 female flies were exposed to 0,6 , or $12 \mathrm{mg} / \mathrm{ml}$ of lamotrigine. There were 4 males and 4 females in each vial, with a total of 40 vials for each treatment. Survivorship was determined with every other day transfers until every fly was dead. A one-way ANOVA and Dunn's multiple comparison tests versus control were used to determine if there were significant differences in mean lifespan between the flies given lamotrigine and the control flies. Additionally, to test for significant differences in maximum lifespan a one-way ANOVA and Dunn's multiple comparison tests versus control were performed using the mean lifespan of the longest-lived ten percent.

\section{Metabolic rate assay}

Control and treated flies were reared for a 10-day period in a similar fashion to that of the mortality assay, as noted above. On day 11, flies were transferred into sex-specific vials containing their respective dosages of lamotrigine. There were 6 vials per dosage, each containing 4 flies. Metabolic rate was evaluated using the Licor LI-6260 gas analyzer. $\mathrm{CO}_{2}$ production in treated flies was compared to that of the control group handled and assayed in parallel. A flow-through respirometer was used to measure the rate of $\mathrm{CO}_{2}$ release from groups of flies following the methods of Williams et al. (2004). Room air was passed through a small chamber containing soda lime, two silica gel chambers, and a Drierite/Ascarite/ Drierite column. This produced a stream of $\mathrm{CO}_{2}{ }^{-}$ free air that was forwarded into the chambers of the flies being measured at a rate of $100 \mathrm{ml} / \mathrm{min}$. The air was directed by a series of computer-controlled valves (Sable Systems, Las Vegas, NV, USA) that allowed separate chambers to be measured in turn. The vials were assayed in a random sequence, allowing for a sampling which was unbiased to temporal and environmental conditions. $\mathrm{CO}_{2}$ levels were averaged and recorded every second using the data acquisition software DATACAN V (Sable Systems). Each vial was measured for $20 \mathrm{~min}$. During periods when they were not being measured, the flies were kept in a flow of dry, $\mathrm{CO}_{2}$-free air by flushing with a separate air stream. The last 5 min of each $20 \mathrm{~min}$ recording of $\mathrm{CO}_{2}$ release was averaged to provide an estimate of relative metabolic rate. The effects of drug treatments on metabolic rates were analyzed with a one-way ANOVA using drug level treated as a fixed effect. 
Locomotion assay

Locomotion studies were conducted to assess the effects of lamotrigine on Drosophila locomotor activity at week one and four of age. Male and female flies were exposed to 0,6 , or $12 \mathrm{mg} / \mathrm{ml}$ of lamotrigine and tested for locomotor activity using the automated Drosophila activity monitor DAM2 (Trikinetics, MA). After 1 and 4 weeks of lamotrigine exposure flies were lightly anesthetized for the purpose of sex classification and to be transferred into the $5 \mathrm{~mm}$ tubes needed for the DAM2 system. To avoid $\mathrm{CO}_{2}$-dependent reductions in locomotor activity as well as to control for any new stimulus that may affect activity, flies were given a 30-min acclimation period prior to be placed in the channels of the monitor. Following the acclimation period data were collected for individual flies at 30-s intervals for a 45-min period resulting in a total of 90 readings per fly.

Any animal with a reading that exceeded the population mean by two SD at any given 30-s interval was excluded from that interval statistical analysis, as abnormally high numbers in activity may have resulted from activity that was localized to the detector beam region. Any animals that had a total number of movements less than two SD from the population mean were excluded, since scores of zero could be due to deaths that occurred in the process of transferring the flies into the $5 \mathrm{~mm}$ tubes.

Repeated measures ANOVA was used to determine significant differences in activity between the three treatment groups. Bonferroni's multiple comparison tests versus control was used to measure significant differences in activity between the control flies and the lamotrigine fed flies.

Fecundity assay

We evaluated age-specific fecundities using the same dosing protocols as above, except that the flies were handled with one female and one male in each assay vial. Fecundity was assayed from day 1 to 10 of adult life. The number of eggs laid each day by each individual female was recorded for this period of 10 days. The impact of drugs on fecundity was assessed by a one-way ANOVA. We also compared the 10-day fecundity of control females to the pooled data from all three doses using a standard $t$-test on the mean values.

\section{Results}

In our dose finding assays, we evaluated the impact of a wide dosing range $(1,3,6,12,24$ and $48 \mathrm{mg} / \mathrm{ml})$ of lamotrigine on fly mortality (data not shown). For both males and females, lamotrigine treatment at 6 or $12 \mathrm{mg} / \mathrm{ml}$ resulted in statistically significant decreases in mortality $(P<0.05$ for both; conducted in duplicate and triplicate, respectively) and $24 \mathrm{mg} / \mathrm{ml}$ of lamotrigine resulted in a statistically significant decrease in mortality for females only $(P<0.05$; conducted in duplicate) (Fig. 1). Lamotrigine was found to be toxic in both males and females at $48 \mathrm{mg} / \mathrm{ml}$. Since 6 and $12 \mathrm{mg} / \mathrm{ml}$ of lamotrigine resulted in the highest mortality reduction in both male and female flies, these doses were chosen to conduct the lifespan assay.

Lamotrigine increased mean lifespan in males and females at both 6 and $12 \mathrm{mg} / \mathrm{ml}$, and it increased maximum lifespan in males and females at $12 \mathrm{mg} / \mathrm{ml}$ (Fig. 2). At $6 \mathrm{mg} / \mathrm{ml}$, mean lifespan was increased by 2.6 days $(12.0 \%)$ in males $(P<0.05)$ and 3.1 days $(12.8 \%)$ in females $(P<0.05)$. At $12 \mathrm{mg} / \mathrm{ml}$, mean and maximum lifespan in males were increased by 3.3 days $(15.4 \%)$ and 6.5 days, respectively; and in females by 4.2 days $(17.2 \%)$ and 6.3 days, respectively $(P<0.01, P<0.001$ for mean increases).

To determine if the observed reduction in mortality was a direct effect of lamotrigine treatment or due to a secondary physiological effect caused by druginduced physiological changes, we examined metabolic rates following lamotrigine treatment. We found

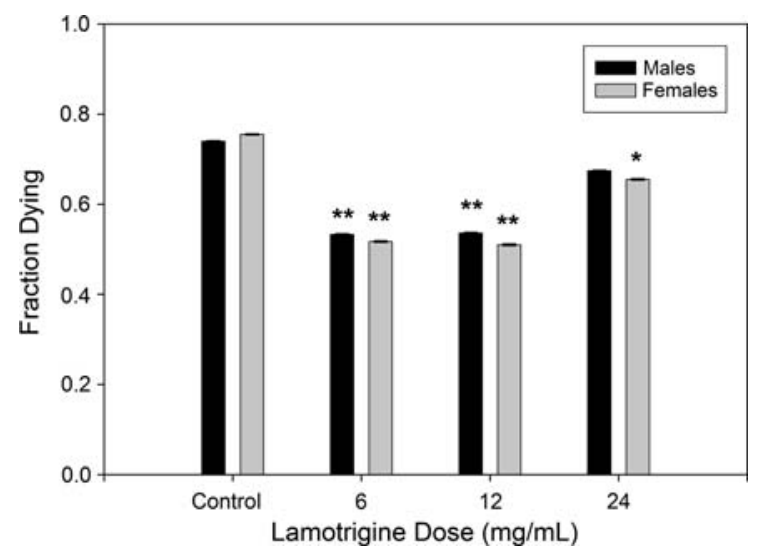

Fig. 1 The effect of lamotrigine on mortality rate at $0,6,12$ and $24 \mathrm{mg} / \mathrm{ml}$. The bars display SEM. * $P<0.01$, ** $P<0.001$ 
Fig. 2 The effect of lamotrigine on the lifespan of female (a) and male (b) flies. In both sexes, lamotrigine resulted in a significant increase in mean lifespan at six $(P<0.05)$ and $12 \mathrm{mg} / \mathrm{ml}(P<0.01)$ and increased maximum lifespan at $12 \mathrm{mg} / \mathrm{ml}$ $(P<0.001)$. Values on the table are means \pm SEM and units are days
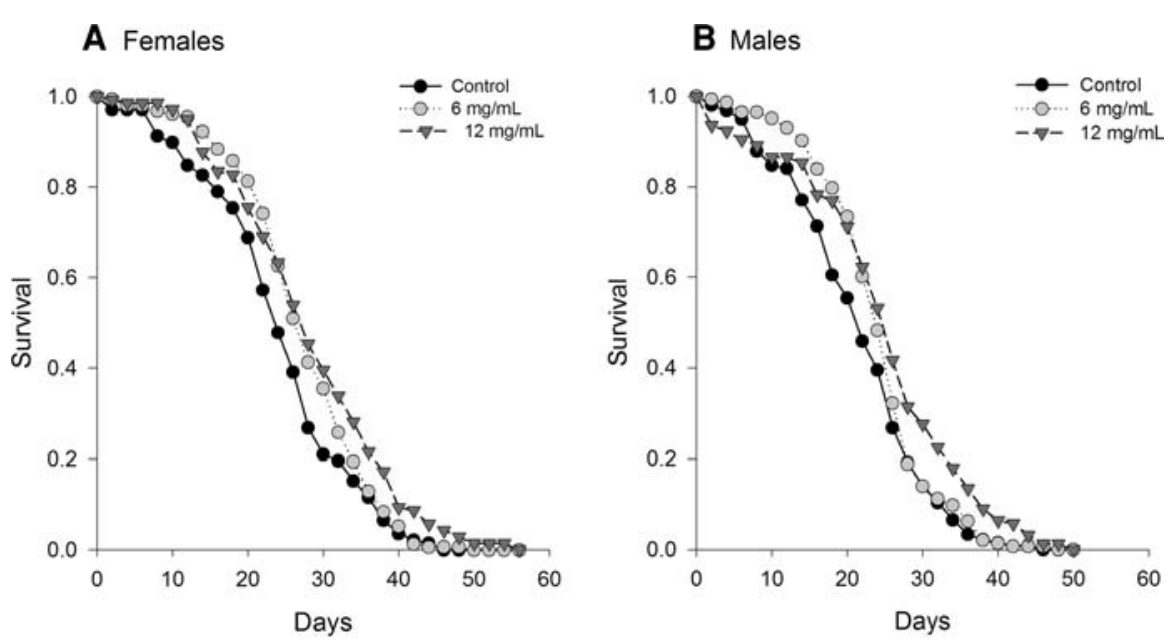

\begin{tabular}{cccccc} 
Sex & Diet & Mean (Days) & P & Maximum (Days) & P \\
\hline Male & control & $21.6 \pm 0.7$ & & $36.8 \pm 0.9$ & \\
Male & $6 \mathrm{mg} / \mathrm{mL}$ & $24.3 \pm 0.6$ & $<0.05$ & $38.4 \pm 0.9$ & $<0.05$ \\
Male & $12 \mathrm{mg} / \mathrm{mL}$ & $24.9 \pm 0.9$ & $<0.01$ & $43.3 \pm 0.9$ & $<0.01$ \\
\hline Female & $\mathrm{control}$ & $24.3 \pm 0.8$ & & $40.7 \pm 0.8$ & \\
Female & $6 \mathrm{mg} / \mathrm{mL}$ & $27.4 \pm 0.7$ & $<0.05$ & $41.3 \pm 0.7$ & $<0.05$ \\
Female & $12 \mathrm{mg} / \mathrm{mL}$ & $28.5 \pm 0.9$ & $<0.001$ & $47.0 \pm 1.3$ & $<0.001$ \\
\hline
\end{tabular}

a trend towards metabolic rate depression in both males and females (Fig. 3). When comparing controls with combined lamotrigine treatment arms, we found that treated males showed a statistically significant depression in metabolic rate $(P<0.05)$ and treated females displayed a trend towards a metabolic rate depression $(P=0.23)$.

Lamotrigine supplemented flies showed a decrease in locomotor activity at week one and four of feeding. At week one, $6 \mathrm{mg} / \mathrm{ml}$ treatment resulted in a $7 \%$ reduction in locomotion in males and a $15 \%$

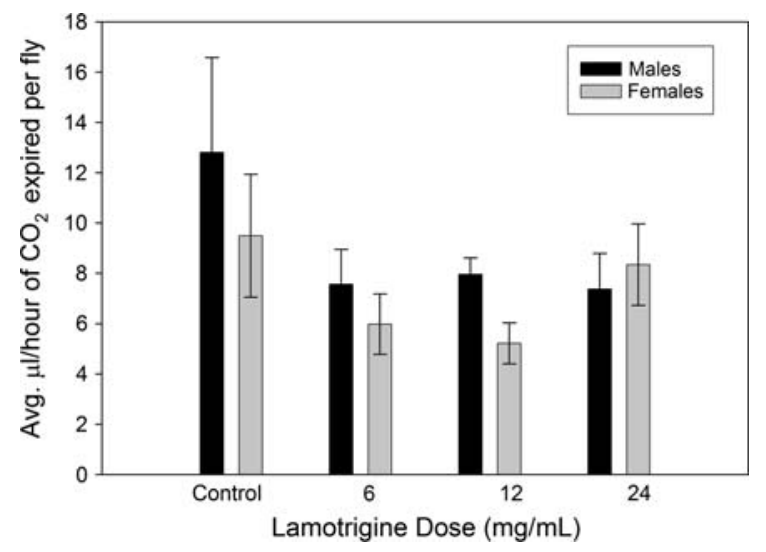

Fig. 3 The impact of $0,6,12$ and $24 \mathrm{mg} / \mathrm{ml}$ of lamotrigine on metabolic rate of adult male and female flies. No statistically significant differences were observed. The bars display SEM reduction in females $(P<0.05$ for both). At week one, $12 \mathrm{mg} / \mathrm{ml}$ treatment caused a $55 \%$ decrease in locomotor activity in females only $(P<0.001)$. At week four, 6 and $12 \mathrm{mg} / \mathrm{ml}$ decreased locomotion in males by 32 and $29 \%$, respectively $(P<0.001$, $P<0.0001)$. At week four, both 6 and $12 \mathrm{mg} / \mathrm{ml}$ caused a $27 \%$ reduction in locomotion in females ( $P<0.01$ for both) (Fig. 4).

It was found that lamotrigine did not impact fecundity, as no significant differences existed between control flies compared to treated flies $(P<$ 0.703).

\section{Discussion}

In our study, the anticonvulsive lamotrigine increased mean and maximum lifespan in male and female Drosophila, but compromised the fly health span, as revealed by a significant decrease in locomotion and a trend towards a depression in metabolic rate. Further, lamotrigine was not found to affect fly fecundity.

Although there are limited studies on anticonvulsants and aging, one report provided evidence that ethosuximide or trimethadione, two commonly prescribed anticonvulsants, increased mean and maximum life-span of C. elegans (Evason et al. 2005). 
Fig. 4 The effect of lamotrigine on the locomotor activity of 1 week old male flies (a), 4 weeks old male flies (b), 1 week old female flies (c), and 4 weeks old female flies (d). * $P<0.05, * *$ $P<0.01, * * * P<0.001$
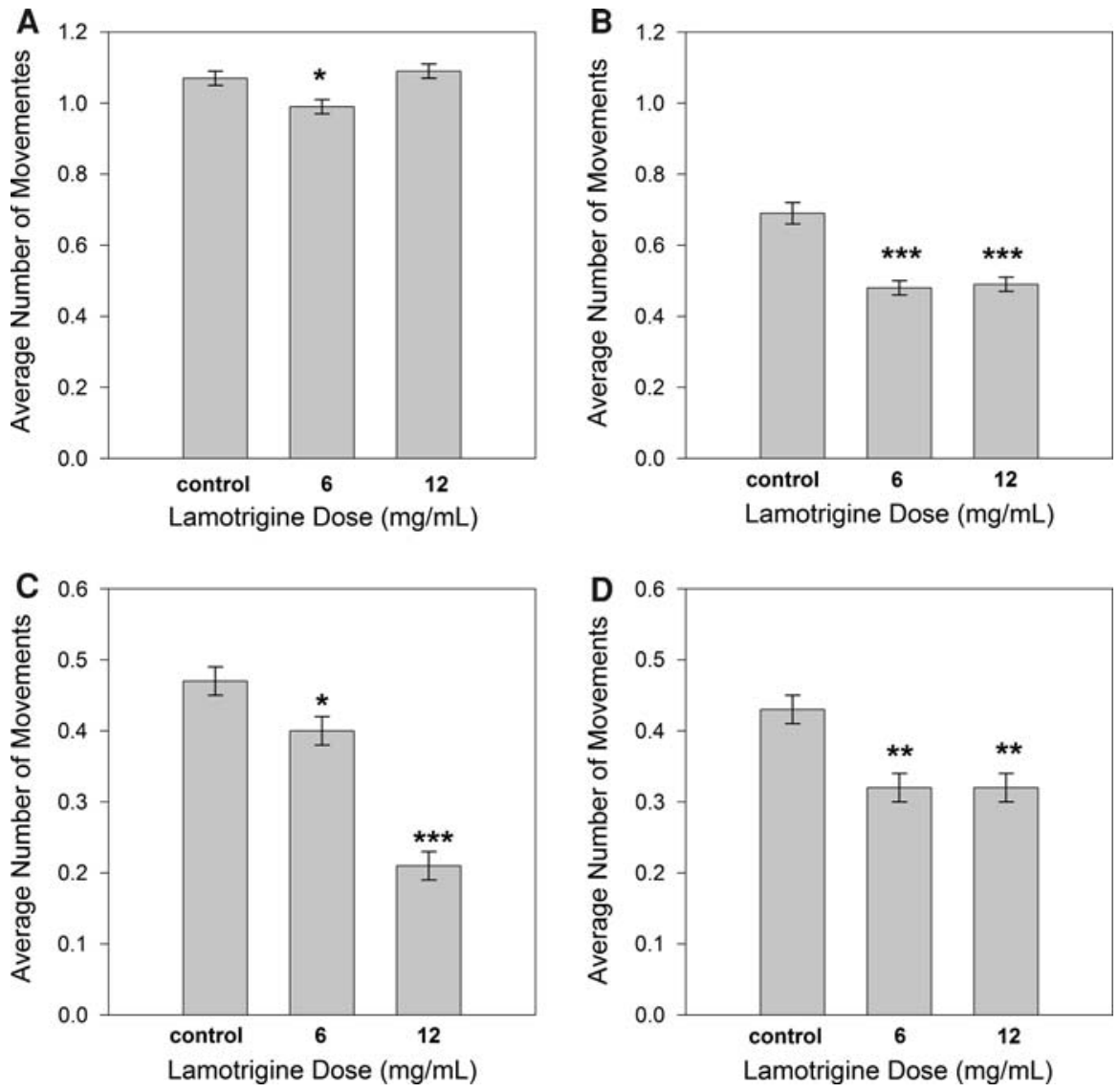

These two drugs have been found to reduce lowthreshold calcium currents in thalamic neurons, which is consistent with their anticonvulsant actions in absence seizures (Coulter et al. 1989). Since most anticonvulsants act as mood stabilizers they may shift C. elegans toward a dauer-like state-a semi-dormant, quiescent state where $C$. elegans decrease their metabolic rate in response to environmental stresses. Therefore, the increased lifespan observed with ethosuximide- and trimethadione-treatment may be due to a decreased metabolic rate.

Lamotrigine anticonvulsive activity is thought to occur through the inhibition of sodium channels, hindering the release of glutamate and aspartate from the axon terminal of neurons (Lang et al. 1993). These neurotransmitters have been implicated in a wide range of physiological functions, such as learning and memory, metabolic processes, and the regulation of respiration, among others (Rock 1968; Fagg and Foster 1983; Wroblewski and Danysz 1989; Advokat and Pellegrin 1992; Navarro et al. 1998;
Burton and Kazemi 2000). The trend in metabolic rate depression that we observed in this study could be due to lamotrigine inhibitory effect on glutamate and aspartate and their roles in respiratory regulation. Although this specific mechanistic hypothesis was not tested in our study, we propose that the neurotransmitters' effect on respiration and their ultimate effect on the metabolic rate could have resulted in lamotrigine-induced life span extension.

It has long been established that reduced metabolic rates can increase poikilotherm life spans. This has been demonstrated in C. elegans and house flies exposed to low temperatures, as well as house flies restricted in activity through the prevention of flight (Ragland and Sohal 1975; Klass 1977; Yan and Sohal 2000). Complementary studies have also shown that increasing the metabolism of honeybees and kestrels results in a decrease in life span (Wolf and SchmidHempel 1989; Daan et al. 1996). In spite of these earlier observations, a recent work reported no correlation between longevity and metabolic rate in 
recombinant inbred Drosophila (Van Voorhies et al. 2003).

Since caloric restriction has been consistently found to increase longevity in mammals and invertebrates, there is the bare possibility that the presence of lamotrigine in the food may induce lower levels of food consumption. This could, in effect, mimic an effect similar to dietary restriction. It is well established that grossly depressed fecundity is associated with dietary restriction sufficient to significantly increase Drosophila longevity (Chippendale et al. 1993). In order to test for possible caloric restriction effects in lamotrigine-supplemented Drosophila, we measured the number of eggs produced over a 10 day period, finding no significant differences in reproductive output when treating with lamotrigine. Since egg production is proportional to food level, the absence of a decline in fecundity indicates that the Drosophila females were not lowering their levels of lamotrigine-supplemented food consumption (Chapman and Partridge 1996). Additionally, the dietary restriction enhancement of longevity observed by Chippendale et al. (1993)using the same type of stock of fruit flies as those used here, required a several-fold reduction in fecundity, we concluded that the extension of life-span which we have observed is not due to a dietary restriction effect mediated by reduced fecundity. Thus, it appears that the effect of lamotrigine on the longevity of adult flies was not mediated solely through effects on some aspect(s) of reproduction.

Since aging is defined by a number of physiological and behavioral changes across the lifespan of an organism, anti-aging compounds should increase lifespan without compromising health span. The association between locomotor activity and aging has been established in a variety of species. A lamotrigineinduced decrease in locomotion is consistent with previous studies. At week one, $6 \mathrm{mg} / \mathrm{ml}$ of lamotrigine resulted in a 7\% reduction in locomotion in males and $15 \%$ in females $(P<0.05)$ and $12 \mathrm{mg} / \mathrm{ml}$ caused a $55 \%$ decrease in locomotor activity in females only $(P<0.001)$. These observations are consistent with the finding that the performance of female flies declines more rapidly with aging (Le Bourg 2004). At week four, 6 and $12 \mathrm{mg} / \mathrm{ml}$ decreased locomotion in males and females to a comparable extent (27$32 \%)$. The significant reduction in locomotion at weeks one and four suggest that anti-aging effects of lamotrigine are associated with compromised function, and in this sense a reduced health span.

In conclusion, the observed decrease in mortality and increase in lifespan as a result of lamotrigine was associated with depression in locomotion and metabolic rate, without a reduction in fecundity. Our results suggest that assessing appropriate indicators of health span is critical in the course of identifying and discovering anti-aging compounds.

Open Access This article is distributed under the terms of the Creative Commons Attribution Noncommercial License which permits any noncommercial use, distribution, and reproduction in any medium, provided the original author(s) and source are credited.

\section{References}

Advokat C, Pellegrin AI (1992) Excitatory amino acids and memory: evidence from research on Alzheimer's disease and behavioral pharmacology. Neurosci Biobehav Rev 1:13-24. doi:10.1016/S0149-7634(05)80046-6

Arking R (1998) Biology of aging: observations and principles. Sinaurer, Sunderland

Burton MD, Kazemi H (2000) Neurotransmitters in central respiratory control. Respir Physiol 2-3:111-121. doi:10. 1016/S0034-5687(00)00153-5

Chapman T, Partridge L (1996) Female fitness in Drosophila melanogaster: an interaction between the effect of nutrition and of encounter rate with males. Proc Biol Sci 1371:755-759. doi:10.1098/rspb.1996.0113

Chippendale AK, Leroi AM, Kim SB, Rose MR (1993) Metabolic aspects of the trade-off between fecundity and longevity in Drosophila malanogaster. J Evol Biol 10:269-293

Clancy DJ, Gems D, Harshman LG, Oldham S, Stocker H, Hafen E, Leevers SJ, Partridge L (2001) Extension of lifespan by loss of CHICO, a Drosophila insulin receptor substrate protein. Science 5514:104-106. doi:10.1126/ science. 1057991

Coulter DA, Huguenard JR, Prince DA (1989) Characterization of ethosuximide reduction of low-threshold calcium current in thalamic neurons. Ann Neurol 6:582-593. doi:10.1002/ana.410250610

Daan S, Deerenberg C, Dijkstra C (1996) Increase daily work precipitates natural death in the kestrel. J Anim Ecol 65:539-544. doi:10.2307/5734

Evason K, Huang C, Yamben I, Covey DF, Kornfeld K (2005) Anticonvulsant medications extend worm life-span. Science 5707:258-262. doi:10.1126/science.1105299

Fagg GE, Foster AC (1983) Amino acid neurotransmitters and their pathways in the mammalian central nervous system. Neuroscience 4:701-719. doi:10.1016/0306-4522 (83)90263-4

Finch CE (1990) Longevity, senescence, and the genome. University of Chicago Press, Chicago 
Jafari M, Rose M (2006) Rules for the use of model organisms in antiaging pharmacology. Aging Cell 5:17-22. doi:10. 1111/j.1474-9726.2006.00195.x

Jafari M, Felgner JS, Khodayari B, Hutchili A, Bussel II, Vince-Cruz C, Rose MR, Mueller LD (2007a) Rhodiola: a promising anti-aging Chinese herb. Rejuvenation Res 4:587-602. doi:10.1089/rej.2007.0560

Jafari M, Khodayari B, Felgner J, Bussel II, Rose MR, Mueller LD (2007b) Pioglitazone: an anti-diabetic compound with anti-aging properties. Biogerontology 6:639-651. doi:10. 1007/s10522-007-9105-7

Jafari M, Zarban A, Pham S, Wang T (2008) Rosa damascena decreased mortality in adult Drosophila. J Med Food 1: 9-13. doi:10.1089/jmf.2007.546

Kang HL, Benzer S, Min KT (2002) Life extension in Drosophila by feeding a drug. Proc Natl Acad Sci USA 2:838-843. doi:10.1073/pnas.022631999

Kenyon C, Chang J, Gensch E, Rudner A, Tabtiang R (1993) A C. elegans mutant that lives twice as long as wild type. Nature 6454:461-464. doi:10.1038/366461a0

Kimura KD, Tissenbaum HA, Liu Y, Ruvkun G (1997) daf-2, an insulin receptor-like gene that regulates longevity and diapause in Caenorhabditis elegans. Science 5328:942946. doi:10.1126/science.277.5328.942

Klass M (1977) Aging in the nematode Caenorhabditis elegans: major biological and environmental factors influencing life span. Mech Ageing Dev 6:413-429. doi:10.1016/0047-6374(77)90043-4

Klass M, Nguyen PN, Dechavigny A (1983) Age-correlated changes in the DNA template in the nematode Caenorhabditis elegans. Mech Ageing Dev 3-4:253-263. doi: 10.1016/0047-6374(83)90080-5

Lang DG, Wang CM, Cooper BR (1993) Lamotrigine, phenytoin and carbamazepine interactions on the sodium current present in N4TG1 mouse neuroblastoma cells. J Pharmacol Exp Ther 2:829-835

Le Bourg E (1987) The rate of living theory. Spontaneous locomotor activity, aging and longevity in Drosophila melanogaster. Exp Gerontol 5:359-369. doi:10.1016/ 0531-5565(87)90034-9

Le Bourg E (2004) Effects of aging on learned suppression of photopositive tendencies in Drosophila melanogaster. Neurobiol Aging 9:1241-1252. doi:10.1016/j.neurobio laging.2003.12.004

Navarro H, Suguihara C, Soliz A, Hehre D, Huang J, Bancalari E (1998) Effect of L-aspartate on the ventilatory response to hypoxia in sedated newborn piglets. Biol Neonate 6: 387-394. doi:10.1159/000014001

Ragland SS, Sohal RS (1975) Ambient temperature, physical activity and aging in the housefly, Musca domestica. Exp Gerontol 5:279-289. doi:10.1016/0531-5565(75)90005-4

Rock G (1968) Nutritional evidence for the absence of the complete ornithine-urea cycle in the insect, Argyrotaenia velutinana (Lepidoptera: torticidae). J Nutr 98:153-158
Rogina B, Reenan RA, Nilsen SP, Helfand SL (2000) Extended life-span conferred by cotransporter gene mutations in Drosophila. Science 5499:2137-2140. doi:10.1126/sci ence.290.5499.2137

Rose (1991) Evolutionary biology of aging. Oxford University Press, New York

Rose MR, Dorey ML, Coyle AM, Service PM (1984) The morphology of postponed senescence in Drosophila melanogaster. Can J Zool 62:1576-1580

Sandstrom DJ (2004) Isoflurane depresses glutamate release by reducing neuronal excitability at the Drosophila neuromuscular junction. J Physiol 558(Pt 2):489-502. doi:10. 1113/jphysiol.2004.065748

Simon AF, Liang DT, Krantz DE (2006) Differential decline in behavioral performance of Drosophila melanogaster with age. Mech Ageing Dev 7:647-651. doi:10.1016/j.mad. 2006.02.006

Smith J (1959) Sex-limited inheritance of longevity in Drosophila subobscura. J Genet 56:1-9. doi:10.1007/ BF02984746

Van Voorhies WA, Ward S (1999) Genetic and environmental conditions that increase longevity in Caenorhabditis elegans decrease metabolic rate. Proc Natl Acad Sci USA 20:11399-11403. doi:10.1073/pnas.96.20.11399

Van Voorhies WA, Khazaeli AA, Curtsinger JW (2003) Selected contribution: long-lived Drosophila melanogaster lines exhibit normal metabolic rates. J Appl Physiol 6:2605-2613

Van Voorhies WA, Curtsinger JW, Rose MR (2006) Do longevity mutants always show trade-offs? Exp Gerontol 10:1055-1058. doi:10.1016/j.exger.2006.05.006

Van Voorhies WA, Melvin RG, Ballard JW, Williams JB (2008) Validation of manometric microrespirometers for measuring oxygen consumption in small arthropods. J Insect Physiol 7:1132-1137. doi:10.1016/j.jinsphys.2008. 04.022

Williams AE, Rose MR, Bradley TJ (2004) The respiratory pattern in Drosophila melanogaster selected for desiccation resistance is not associated with the observed evolution of decreased locomotory activity. Physiol Biochem Zool 1:10-17. doi:10.1086/381467

Wolf T, Schmid-Hempel P (1989) Extra loads and foraging lifespan in honeybee workers. J Anim Ecol 58:943-954. doi: $10.2307 / 5134$

Wroblewski JT, Danysz W (1989) Modulation of glutamate receptors: molecular mechanisms and functional implications. Annu Rev Pharmacol Toxicol 29:441-474. doi:10.1146/annurev.pa.29.040189.002301

Yan LJ, Sohal RS (2000) Prevention of flight activity prolongs the life span of the housefly, Musca domestica, and attenuates the age-associated oxidative damage to specific mitochondrial proteins. Free Radic Biol Med 11:11431150. doi:10.1016/S0891-5849(00)00423-8 